\title{
Progression of impending retinal vein occlusion to the ischemic variant following intravitreal ranibizumab
}

\author{
Reem Z. Renno, $\mathrm{MD}^{1,2 *}$ \\ ${ }^{1}$ Methodist Willowbrook Hospital, Houston, Texas, USA \\ ${ }^{2}$ North Cypress Medical Center, Houston, Texas, USA
}

\begin{abstract}
An 82-year-old woman who had experienced two episodes of amaurosis fugax in her left eye presented with vision loss. She was diagnosed with impending retinal vein occlusion (RVO) of the left eye and received an intravitreal injection of ranibizumab. One month after this injection she was diagnosed with ischemic RVO.

At 8-weeks post-presentation, extremely ischemic features were observed of severe vascular attenuation and extensive retinal capillary obliteration. She experienced no visual improvement over the following several months.
\end{abstract}

We report a case of ischemic retinal vein occlusion (RVO) in a patient who initially had impending RVO and who was subsequently treated with intravitreal ranibizumab and progressed to ischemic RVO despite treatment.

\section{Case report}

An 82-year-old woman with a history of well-controlled ocular hypertension (using dorzolamide-timolol $0.5 \%$ drops) presented because of vision loss in her left eye. Four weeks prior to her first visit to our clinic she had noted a sudden blurring over the entire visual field of her left eye. This lasted for approximately ten minutes and then fully recovered; eight days later, she experienced exactly the same phenomenon. Her previous clinical history was only significant for well- controlled hypertension (on lisinopril and baby aspirin).

On examination, small optic nerve head, mild dilatation and tortuosity of the retinal veins, and some intraretinal hemorrhages were observed at the posterior pole (Figure 1). Her best- corrected visual acuity (BCVA) was 20/80, and her intraocular pressure (IOP) was 22 mmHg. Fluorescein angiography (FAG) revealed normal choroidal filling and slightly delayed arterio-venous filling without capillary nonperfusion. Optical coherence tomography revealed increased retinal thickness in the macular area. The right eye was normal. She was diagnosed with impending CRVO and underwent a trial of intravitreal ranibizumab $(0.5 \mathrm{mg}$ in $0.1 \mathrm{~mL})$. Laboratory data were unremarkable, including hemoglobin, platelet count, white cell count, electrolytes, and lipid profile. Additional examinations were subsequently performed; inflammatory markers and plasma viscosity were unremarkable. Carotid duplex ultrasound showed no significant stenosis in the ipsilateral carotid artery.

At Four-weeks post-presentation, the patient noted a significant decrease in visual acuity; her BCVA had declined to count fingers. Fundus examination revealed numerous retinal hemorrhages in four quadrants, dilated and tortuous retinal veins, and flame shaped hemorrhage at disc. Optical coherence tomography revealed improved retinal thickness in the macular area. Patient received a second dose of ranibizumab. Progression of retinal vein occlusion was assumed.

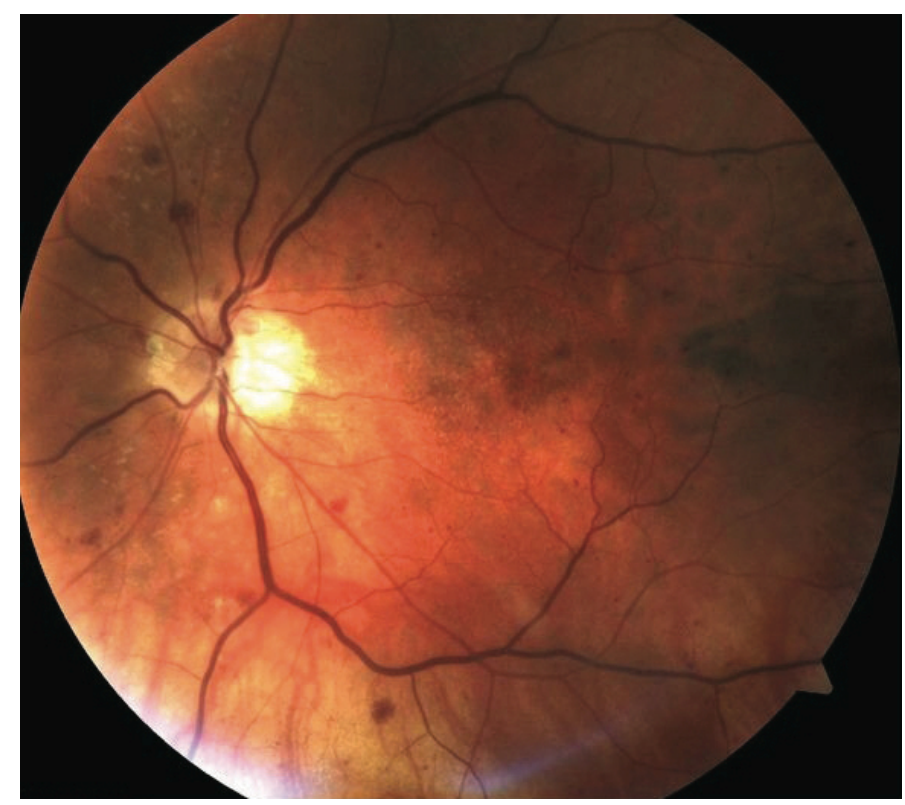

Figure 1. Fundus photo of the left eye at presentation: Dilated and tortuous retinal veins, some intraretinal hemorrhages at the posterior pole

At follow up eight weeks later, her vision remained same and fundus examination revealed reductions in retinal hemorrhage and cotton-wool spots, moderate macular edema, severe attenuation of the retinal vessels and a somewhat sclerotic retinal vasculature in addition to some optic nerve pallor (Figure 2). A full blown ischemic CRVO

*Correspondence to: Reem Z. Renno, MD, 13300 Dotson Rd, Houston, Texas 77065, USA. Tel: 832-492-7248; E-mail: reem_renno@yahoo.com

Key words: ranibizumab, impending central retinal vein occlusion

Received: April 05, 2018; Accepted: April 21, 2018; Published: April 25, 2018 


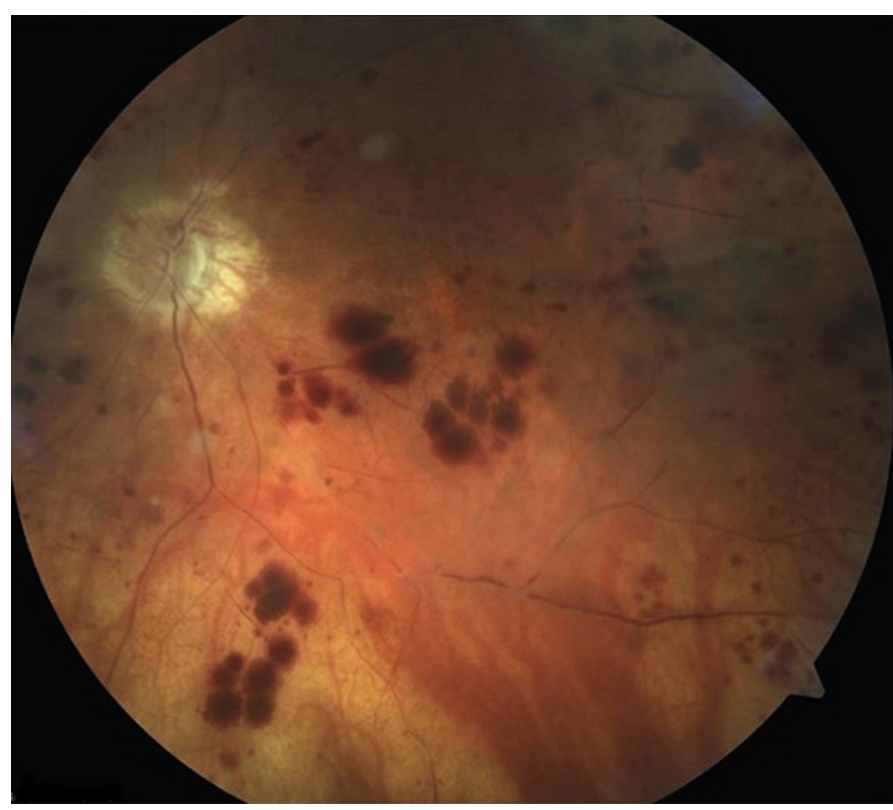

Figure 2. The left eye at twelve-weeks post-presentation: extensive retinal capillary obliteration and severe attenuation of the retinal vessels; more diffuse retinal hemorrhages and optic disc pallor.

was diagnosed and continued to observe the patient without additional treatment: Her left eye showed minimal visual recovery to a BCVA 20/400.

\section{Discussion}

We report a case of ischemic CRVO that initially presented as impending CRVO. Impending CRVO is an uncommon condition, which may resolve or progress to complete obstruction [1]. Our patient experienced recurrent amaurosis fugax in the setting of slightly dilated and tortuous retinal veins and scattered flame-shaped hemorrhages as signs of future CRVO that became ophthalmoscopically evident within four weeks of the onset of symptoms.
The patient was given one intravitreal injection of ranibizumab $(0.5 \mathrm{mg})$ for macular edema. Four weeks after this injection, her BCVA abruptly decreased to counting fingers. It ultimately stabilized at BCVA of $20 / 400$.

Anti-vascular endothelial growth factor treatment is known to minimize macular edema in the setting of central retinal vein occlusion. However, it does not stop the progression to ischemic CRVO: whether it worsens the course of the condition is unclear though.

Vascular endothelial growth factor (VEGF) is known to have a neurotrophic effect, and blockage of all VEGF isoforms by bevacizumab may create toxic effects [2]. Neuronal tissue, including the neurosensory retina, may be vulnerable to complete VEGF inhibition, especially in the presence of a disease like CRVO. A recent case report of ischemic CRVO which progressed from nonischemic CRVO following intravitreal bevacizumab injection supports this hypothesis [3]. Additionally, the fact that our patient had small optic nerve heads and concomitant ocular hypertension could have contributed negatively to the progression.

Further work to identify factors involved in mediating retinal ischemia deserved being pursued to help device more rational interventional approaches to RVO treatment at different stages.

\section{Acknowledgments}

The author wishes to thank Nina Mussa for her assistance in preparing the figures.

\section{References}

1. Gass JD. Stereoscopic atlas of macular diseases: diagnosis and treatment. 4th ed. St Louis: Mosby; 1997. Page 546-555.

2. Kim KS, Chang HR, Song S (2008) Ischaemic change after intra-vitreal bevacizumab (Avastin) injection for macular oedema secondary to non-ischemic central retinal vein occlusion. Acta Ophthalmol 86: 925-927. [Crossref]

3. Na Rae Kim and Hee Seung Chin (2010) Progression of Impending Central Retinal Vein Occlusion to the Ischemic Variant Following Intravitreal Bevacizumab. Korean $J$ Ophthalmol 24: 179-181. [Crossref]

Copyright: (C2018 Renno RZ. This is an open-access article distributed under the terms of the Creative Commons Attribution License, which permits unrestricted use, distribution, and reproduction in any medium, provided the original author and source are credited. 